\title{
Interaction of nitrogen ions with beryllium surfaces
}

Katharina Dobes ${ }^{1}$, Martin Köppen ${ }^{2}$, Martin Oberkofler ${ }^{3}$, Cristian P. Lungu ${ }^{4}$, Corneliu Porosnicu ${ }^{4}$, Till Höschen ${ }^{3}$, Christian Linsmeier ${ }^{2}$, and Friedrich Aumayr ${ }^{{ }^{*}}$

${ }^{1}$ Institute of Applied Physics, TU Wien, Association EURATOM ÖAW, Vienna Austria ${ }^{2}$ Institute of Energy and Climate Research - Plasma Physics, Forschungszentrum Jülich GmbH, Association EURATOM-FZJ, 52425 Jülich, Germany

${ }^{3}$ Max-Planck-Institut für Plasmaphysik, EURATOM Association, 85748 Garching, Germany

${ }^{4}$ National Institute for Laser, Plasma, and Radiation Physics, Bucharest, Romania

\begin{abstract}
The interaction of energetic nitrogen projectiles with a beryllium surface is studied using a highly sensitive quartz crystal microbalance technique. The overall mass change rate of the beryllium sample under $\mathrm{N}_{2}{ }^{+}$ion impact at an ion energy of $5000 \mathrm{eV}$ (i.e. $2500 \mathrm{eV}$ per $\mathrm{N}$ ) is investigated in situ and in real-time. A strong dependency of the observed mass change rate on the nitrogen fluence (at constant flux) is found and can be attributed to the formation of a nitrogen containing mixed material layer within the ion penetration depth. The presented data elucidate the dynamics of the interaction process and the surface saturation with increasing nitrogen fluence in a unique way. Basically, three distinct interaction regimes can be discriminated, which can be linked to the evolution of the surface composition upon nitrogen impact.

Steady state surface conditions are obtained at a total cumulative nitrogen fluence of $\sim 8.3 \cdot 10^{17} \mathrm{~N}$ atoms per $\mathrm{cm}^{2}$. In dynamic equilibrium, the interaction is marked by continuous surface erosion. In this case, the observed total sputtering yield becomes independent from the applied nitrogen fluence and is of the order of 0.4 beryllium atoms per impinging nitrogen atom.
\end{abstract}

Keywords: plasma wall interaction, sputtering, erosion, beryllium

*aumayr@iap.tuwien.ac.at 


\section{Introduction}

The international experimental thermonuclear fusion reactor ITER is currently being built in the south of France. Its aim is to demonstrate the usability of fusion for power generation. ITER will be the first machine to ever deliver a net energy output, with a scientific goal of producing ten times the power that is needed as auxiliary heating power in order to sustain the fusion reaction (i.e. a fusion power gain of $Q \sim 10$ ).

As a result of the rather extreme conditions, which will be necessary to initiate the fusion reaction in a future fusion power plant, one of the greatest challenges in the successful realization of the ITER project will be the interaction of the very hot fusion plasma with the walls of its vacuum vessel. A profound knowledge on the key plasma-wall interaction issues such as surface erosion, implantation of impurities and retention of fueling hydrogen isotopes is therefore strongly desired.

In ITER a material mix of beryllium on the first wall and tungsten in the divertor at the bottom of the machine is envisaged. ASDEX Upgrade was the first fusion machine to operate with a full tungsten wall [1]. With the transition to all-metal machines, the intrinsic impurity levels (of. e.g. carbon) in the plasma edge decreases considerably and their beneficial influence on heat load mitigation by radiative cooling is reduced [2]. In order to maintain the desired radiated power fraction in fusion machines such as ITER, a controlled injection of impurity species to replace intrinsically available coolants will therefore be mandatory [3]. For this purpose, nitrogen has proven to be a valuable substitute for e.g. carbon in the divertor region, due to its comparably high radiative characteristics at low electron temperatures [1,4]. Nitrogen seeding with feedback control has already matured into a standard operational scenario in today's fusion experiments such as ASDEX Upgrade [2] or JET [5,6]. With nitrogen as a seeding gas, the divertor target power flux could be mitigated by high radiation to a technically acceptable level, and the plasma performance could even be increased compared to discharges without impurity seeding [1]. In a series of recent laboratory experiments it was observed that under nitrogen impact, a stable nitride phase is formed within the penetration range in both ITER plasma wall materials, i.e. a WN [7] compound and $\mathrm{Be}_{3} \mathrm{~N}_{2}$, respectively [8]. 
Compositional changes of the plasma facing components might have a severe influence on material properties such as the sputtering yield, the melting point, the fuel retention behavior or the electrical conductivity of the surface. The evolution of plasma facing materials like tungsten and beryllium in the environment of a burning fusion plasma, especially the mixing with impurity and fueling species, is therefore of considerable interest.

In this work the saturation dynamics of a beryllium surface under nitrogen impact was studied in detail. The investigations were performed under controlled laboratory conditions using a mono-energetic nitrogen ion beam. Mass change rates were determined in situ and in real-time.

A unique insight into the dynamics and built-up of mixed nitrogen containing beryllium layers could be gained, which can be a valuable contribution for understanding the basic mechanisms of the involved processes. In addition steady state surface conditions were also studied, where the simultaneous formation and erosion of the resulting modified surface layer is in dynamic equilibrium.

\section{Experimental approach}

Total mass change rates of a beryllium surface under nitrogen impact were studied at IPP Garching using a highly sensitive quartz crystal microbalance technique developed at the University of Technology in Vienna [9-11]. The centerpiece of the setup is a stress compensated (SC) cut quartz crystal, which is driven at its thickness shear mode in series resonance at a resonance frequency of about $6 \mathrm{MHz}$.

One side of the quartz crystal is coated with a $500 \mathrm{~nm}$ thick beryllium layer. The beryllium layers are fabricated by thermionic vacuum arc deposition at the National Institute of Laser, Plasma and Radiation Physics in Bucharest [12]. Dense, bulk like beryllium layers are produced by accelerating the beryllium ions onto the gold substrate by means of a negative bias of - $750 \mathrm{~V}$. The substrate temperature during deposition is kept at $473 \mathrm{~K}$. The beryllium films are transferred in air before being installed in a vacuum chamber for exposure to a 
nitrogen ion beam. The beryllium samples used in the presented studies therefore exhibit a native oxide layer on the surface.

Total mass changes of the target film under nitrogen impact are determined from a change in the resonance frequency of the quartz crystal according to the relation $\Delta f / f=-\Delta m / m[13]$. In our experiments any mass change rate is therefore derived from the slope of a linear fit to the frequency-change curve with fluence. In cases where the mass change rate changes with the cumulative ion fluence, a step-wise linear approximation is used to evaluate the mass change rate of the target film.

The QCM technique exploited in our investigations is by design only capable of detecting total mass changes, but does not deliver any information on the actually sputtered species. In addition, any simultaneous mass increase $+\Delta m$ (due to e.g. projectile implantation) and mass loss $-\Delta m$ (by e.g. material erosion) cannot be distinguished. Thus only the balance of all processes, which result in a mass change of the target film, is observable.

The quartz crystal is mounted on a sample holder, which is depicted in figure 1. The beryllium-coated quartz (1) is clamped onto the sample holder by a tungsten spring from the back (2) and with a gold ring from the front (3), in order to avoid mechanically induced stress. The sample holder is equipped with a faraday cup (4) for frequent ion current density determination in between surface exposures to the impinging nitrogen beam. A linear translation stage allows for changing between quartz and faraday cup position.

Experiments are performed in an ultra high vacuum chamber at a base pressure of approximately $1 \cdot 10^{-9}$ mbar. The chamber is pumped by a combination of a turbo-molecular pump, a titanium sublimation pump and a cryo-pump, which is cooled with liquid nitrogen during the experiments.

In order to minimize influences of temperature changes during ion bombardment on the resonance frequency of the quartz crystal, measurements were always conducted at an elevated surface temperature of $465 \mathrm{~K}$, which is the temperature where the resonance frequency of the quartz is least sensitive to temperature fluctuations. The crystal is heated with a constant heating power by means of a coaxial heater (5) in figure 1), clamped onto the sample holder. The temperature is monitored by a k-type thermocouple (6). A sophisticated 
electronics permits the detection of mass changes of as small as 0.2 $\mathrm{amu} / \mathrm{ion} \cdot \mathrm{nA} / \mathrm{cm}^{2}[10,11]$, corresponding to approximately $5 \cdot 10^{-5}$ beryllium monolayers per second for typical ion current densities characteristic to the experimental conditions in the presented investigations.

A schematics of the experimental setup is shown in figure 2. Molecular $\mathrm{N}_{2}{ }^{+}$ions are produced in a commercially available electron impact ion source (SPECS IQE 12/38). The source is operated at a pressure of $\sim 1-5 \cdot 10^{-5}$ mbar, which results in a pressure increase to about $10^{-7}$ mbar in the experimental chamber during source operation.

A Wien velocity filter is used to select the molecular ions from the extracted ion beam. The ion source and the direction of the ion beam when it exits the Wien filter, are slightly off axis in order to keep neutral particles from passing the setup. The ions are accelerated from the ion source with an extraction voltage of $5000 \mathrm{~V}$, resulting in a surface impact energy of $2500 \mathrm{eV}$ per constituent nitrogen atom. By means of two lenses and a pair of deflection plates, the extracted ion beam is then guided and focused onto the sample position in the experimental chamber.

The oscillation amplitude of the quartz decreases from the center outwards, which introduces a radial dependency of the sensitivity of the quartz to mass changes (energy trapping [13]). To eliminate this effect in the determination of mass change rates from the observed frequency evolution of the quartz, the whole mass sensitive inner part of the crystal (the so-called active area) has to be covered with a homogenous ion current density. Therefore the impinging ion beam is scanned rapidly across the entire active area by applying two AC voltages of different frequency to a pair of deflection plates just in front of the quartz crystal (cf. figure 2). Current density variations within the active area were always kept below $\sim 10 \%$, to enable an accurate determination of the impinging ion flux covering the sample area. In doing so mechanical stress imposed on the crystal by non-uniform mass erosion is additionally avoided. 


\section{Results and discussion}

The evolution of the mass change rate per incident nitrogen atom of a beryllium surface as a function of the applied cumulative nitrogen atom fluence was determined for $\mathrm{N}_{2}{ }^{+}$impact at a total kinetic energy of $5000 \mathrm{eV}$ (i.e. $2500 \mathrm{eV}$ per $\mathrm{N}$ ). The frequency evolution of the quartz crystal as a function of the applied $\mathrm{N}$ fluence is shown in figure 3 . The corresponding mass change rate derived from it, is shown in figure 4. The two curves summarize the nitrogen saturation behavior of the beryllium sample from an as-received to a nitrogen saturated surface, with a steady state surface composition.

It was found that the observed mass change rate depends strongly on the applied ion fluence (at constant flux), which is most significant for low nitrogen fluences. This dynamics can be ascribed to the formation of beryllium nitride within the ion penetration range as it has been observed in recent studies [8].

For low fluences $\Phi \lesssim 4 \cdot 10^{15} \mathrm{~N} / \mathrm{cm}^{2}$, the interaction of the nitrogen projectiles with the beryllium surface is at first dominated by a high net erosion rate. This initially very pronounced mass removal rate, slows down quickly with increasing fluence and can most likely be attributed to the removal of loosely bound surface adsorbates from the fresh beryllium surface, such as a native beryllium-oxide layer from transport and storage of the samples under ambient conditions or some carbon contaminants.

Essentially, the following nitrogen saturation dynamics can be sectioned into three different sub-parts, as also indicated in figure 4:

(1) $0.4 \cdot 10^{16} \lesssim \Phi \lesssim 12 \cdot 10^{16} \mathrm{~N} / \mathrm{cm}^{2}$ a regime of net mass increase (i.e. a positive $\Delta m$ in figure 4).

(2) $12 \cdot 10^{16} \lesssim \Phi \lesssim 83 \cdot 10^{16} \mathrm{~N} / \mathrm{cm}^{2}$ a regime of net mass loss, where the observed erosion rate still changes with the ion fluence (i.e. a negative $\Delta m$ in figure 4).

(3) $\Phi \gtrsim 8.3 \cdot 10^{17} \mathrm{~N} / \mathrm{cm}^{2}$ steady state erosion, where the observed erosion rate furthermore remains constant with increasing fluence. 
The total observed mass change rate represents the balance of different processes, which occur simultaneously as the surface is bombarded with nitrogen projectiles. Under nitrogen impact, an altered surface layer builds on top of the beryllium substrate. The composition of this surface layer will dynamically change during nitrogen bombardment, until steady state surface conditions are established at a cumulative ion fluence of $\Phi \sim 8.3 \cdot 10^{17} \mathrm{~N} / \mathrm{cm}^{2}$ and the observed mass change rate becomes constant.

The three above-sketched sub-sections of this transient behavior of the mass change rate, reflect this saturation dynamics and can be qualitatively understood in the following way:

Impinging nitrogen projectiles are implanted at their ion penetration depth. It was observed [8] that the thickness of the beryllium nitride layer, which builds, when a beryllium surface is bombarded with energetic nitrogen atoms, is of the order of the ion penetration depth in the surface. From this it can be inferred that generally the majority of projectiles will remain at the depth at which they come to rest in the surface and do not diffuse much beyond the implantation zone into the underlying beryllium bulk. For a limited projectile diffusivity, this will result in the built-up of a nitrogen containing, mixed material layer with increasing projectile fluence, which is localized around the ion penetration depth below the surface.

In addition material is simultaneously eroded from the surface. As the nitrogen concentration in the surface increases with applied ion fluence, both the implanted nitrogen atoms as well as the beryllium substrate will be sputtered. The partial sputtering yield of the two species will depend on their individual near surface concentrations, as eroded particles predominantly originate from within only a very shallow sub-surface region [14].

By continuous surface erosion and simultaneous implantation of projectiles at their penetration depth, the nitrogen containing surface layer will broaden and the profile of implanted nitrogen will increasingly extend towards the surface until finally, implanted nitrogen projectiles will emerge into the near surface zone, from which sputtered atoms originate. This will eventually result in an increased partial nitrogen sputtering yield. 
Finally, after a surface layer with a thickness of the order of the ion penetration depth has been eroded, dynamic equilibrium conditions for simultaneous nitrogen implantation and erosion are established. At this point the nitrogen saturated surface zone extends from the nitrogen penetration depth to the surface and the total amount of retained nitrogen saturates. Additional ion bombardment has no further effect on the surface composition or the observed mass change rate.

In the regime of net mass increase (1) in figure 4), the mass change due to projectile implantation exceeds the overall mass loss due to surface erosion. At a fluence of $\sim 1.2 \cdot 10^{17} \mathrm{~N}$ per $\mathrm{cm}^{2}$ a transition to net erosion is observed (2). This is most likely linked to obtaining the local nitrogen saturation concentration at the ion penetration depth, which results in a reduction of the projectile implantation rate as excess nitrogen is implanted less efficiently and effuses out of the surface. As a result, the mass loss due to surface erosion at some point starts to dominate the observed mass change. However, for fluences between $1.2 \cdot 10^{17} \lesssim \Phi \lesssim$ $8.3 \cdot 10^{17} \mathrm{~N}$ per $\mathrm{cm}^{2}$ the observed erosion rate still increases slightly with applied nitrogen fluence.

Steady state surface conditions, i.e. an erosion rate which furthermore remains constant, are only reached at a total fluence of $\sim 8.3 \cdot 10^{17} \mathrm{~N}$ per $\mathrm{cm}^{2}$ (3). In steady state the nitrogen content in the surface becomes constant, i.e. the erosion of nitrogen from the surface is in dynamic equilibrium with nitrogen implantation. For fluences of $\Phi \gtrsim 8.3 \cdot 10^{17} \mathrm{~N}$ per $\mathrm{cm}^{2}$ the observed mass loss rate can therefore be attributed to beryllium sputtering only. It amounts to $\sim 0.4$ beryllium atoms being sputtered per impinging nitrogen atom.

Although the local nitrogen concentration at the implantation depth saturates already at a fluence of $\sim 1.2 \cdot 10^{17} \mathrm{~N}$ per $\mathrm{cm}^{2}$, it might take much longer until the resulting nitrogen saturated sub-surface zone emerges into the shallow escape depth of sputtered particles by continuous surface recession due to erosion. For limited nitrogen diffusivity, it can be expected that a surface layer as thick as the ion penetration range has to be eroded to establish steady state surface conditions.

The nitrogen range in beryllium at an impact energy of $2500 \mathrm{eV}$ can be estimated from a simulation with the Monte Carlo code TRIDYN [15], which does a binary 
collision approximation for the slowing down of the projectile and the recoiling target atoms in the collision cascade, which is initiated upon energetic particle impact. From a 'static' simulation, where any compositional changes to the surface during ion bombardment are suppressed, it can be expected that the most probable implantation depth of the nitrogen projectiles is at $\sim 87 \AA$ below the surface. From the experimental data on the other hand, the surface recession can be estimated by integrating the observed mass change rate in figure 4 . At the fluence at which steady state surface conditions are reached (i.e. 8.3.1017 $\mathrm{N}$ per $\mathrm{cm}^{2}$ ), the thus obtained total surface recession is $\sim 117 \AA$. From this simple estimation it is concluded, that indeed a surface layer with a thickness slightly exceeding the ion penetration depth is eroded, before steady state surface conditions are obtained in the experiment.

We have not taken into account that in addition the continuous exposure of the beryllium sample to the nitrogen beam might result in a roughening of the surface. This will generally lead to an increased sputtering yield [14] and might therefore also contribute to the observed erosion dynamics in the sputtering dominated regime (2). To this point the experimentally available data does not suffices to further assess to which extend the saturation dynamics of the surface reflects the evolution of the surface composition on the one hand and any possible alteration of the surface morphology on the other hand.

\section{Summary \& conclusion}

The interaction of energetic nitrogen projectiles with a beryllium surface was studied in situ and in real-time using a highly sensitive quartz crystal microbalance technique. It was found that the observed mass change rate depends strongly on the applied ion fluence. A unique insight into the dynamics and built-up of a nitrogen containing surface layer could be gained. It was found that the saturation dynamics is mainly composed of three individual regimes: at first a regime of dominant projectile implantation at the ion penetration depth (and hence a net mass increase). As the nitrogen content at the implantation depth saturates and the implantation probability is consequently reduced, a transition to net erosion is observed. In the sputtering dominated regime a continuous increase in the total sputtering yield with increasing cumulative 
nitrogen fluence is observed. Both, the evolution of the surface composition in the near surface escape depth of sputtered particles as well as an alteration of the surface morphology might contribute to this behavior.

Dynamic equilibrium is established at a total fluence of $8.3 \cdot 10^{17} \mathrm{~N}$ per $\mathrm{cm}^{2}$, after a surface layer with a thickness exceeding the ion penetration depth estimated with TRIDYN is removed. In this case the nitrogen content at the implantation depth as well as in the escape zone of sputtered particles becomes constant and the observed net mass change rate per impinging ion is furthermore independent from the applied ion fluence. In steady state a net sputtering yield of 0.4 beryllium atoms per impinging nitrogen atom is observed.

\section{Acknowledgements}

This work was supported by the European Communities under the Contract of Association between EURATOM and the Austrian Academy of Science. It was carried out within the framework of the European Fusion Development Agreement as part of the EFDA Task Force on Plasma Wall Interactions. The views and opinions expressed herein do not necessarily reflect those of the European Commission. 


\section{References}

[1] A. Kallenbach, R. Dux, J.C. Fuchs et al., Plasma Phys. Control. Fusion 52 (2010), 055002

[2] A. Kallenbach, R. Dux, M. Mayer et al., Nucl. Fusion 49 (2009), 045007

[3] J. Rapp, G.F. Matthews, P. Monier-Garbet et al., J. Nucl. Mat. 337 - 339 (2005), 826

[4] A. Kallenbach, M. Balden, R. Dux et al., J. Nucl. Mat. 415 (2011), S19

[5] R. Neu, G. Arnoux, M. Beurskens et al., Phys. Plasmas 20 (2013), 056111

[6] M. Oberkofler, D. Douai, S. Brezinsek et al., J. Nucl. Mat. 438 (2013), S258

[7] K. Schmid, A Manhard, Ch. Linsmeier et al., Nucl. Fusion 50 (2010), 025006

[8] M. Oberkofler and Ch. Linsmeier, Nucl. Fusion 50 (2010), 125001

[9] T. Neidhart, Z. Toth, M. Schmid, and P. Varga, Nucl. Instrum. and Meth. B 90 (1994), 496

[10] G. Hayderer, M. Schmid, P. Varga, HP. Winter and F. Aumayr, Rev. Sci. Instrum. 70 (1999), 3696

[11] A. Golczewski, K. Dobes, G. Wachter, M. Schmid and F. Aumayr, Nucl. Instrum. and Meth. B 267 (2009), 695

[12] C. P. Lungu, I. Mustata, V. Zaroschi, A. M. Lungu, P. Chiru, M. Rubel, P. Coad, and G.F. Matthews, Phys. Scr. T 128 (2007), 157

[13] G. Sauerbrey, Z. Phys. 155 (1959), 206

[14] P. Sigmund, Phys. Rev. 184 (1969), 383

[15] W. Möller and W. Eckstein, Nucl. Instrum and Meth. B 2 (1984), 814 


\section{Captions}

Figure 1: Sketch of the sample holder, including: the quartz crystal (1), the crystal clamping from the back with a W spring (2) and from the front with a $\mathrm{Au}$ ring (3), the Faraday cup (4) for ion current density determination a coaxial heater (5) and thermocouple (6) to keep and monitor the quartz operating temperature.

Figure 2: Schematics of the experimental setup (not to be scaled). Ions are produced in an electron impact ion source, selected according to their mass in a Wien filter and then focused and deflected onto the sample position by means of a lens system and deflection plates. Mass change rates are determined with a quartz crystal microbalance.

Figure 3: Evolution of the quartz frequency as a function of the applied $\mathrm{N}$ fluence observed when saturating a virgin beryllium surface with $\mathrm{N}_{2}{ }^{+}$ions at an impact energy of $5000 \mathrm{eV}(2500 \mathrm{eV}$ per $\mathrm{N})$.

Figure 4: Evolution of the total mass change rate of the beryllium surface as a function of the applied $\mathrm{N}$ fluence. The dynamic behavior can be sectioned into different sub-parts. After the removal of loosely bound surface adsorbates, (1) an implantation dominated and (2) an erosion dominated regime are observed, until finally (3) steady state conditions are established. For details see text. 
Figures

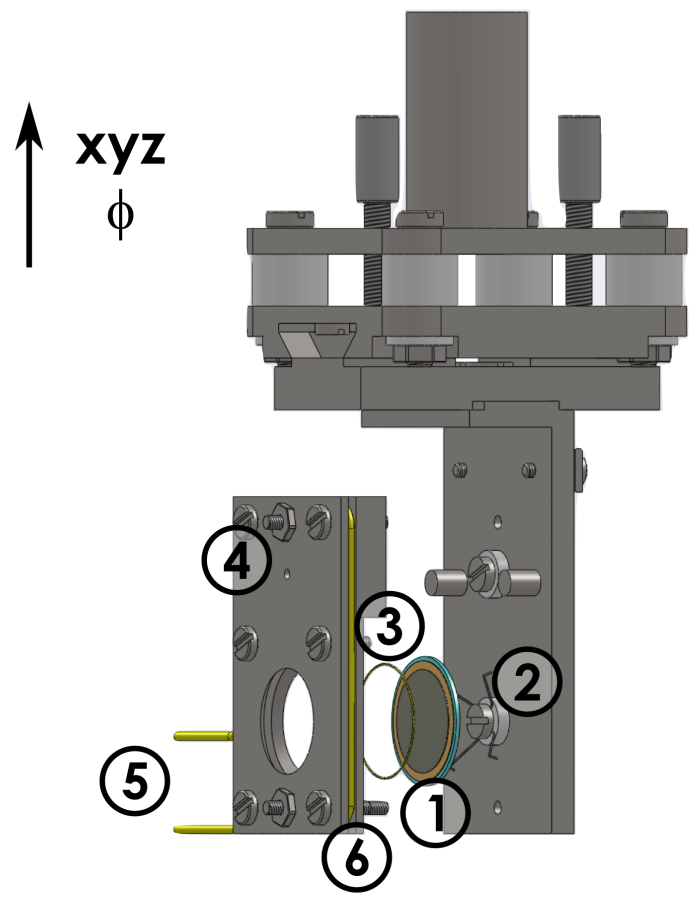

Figure 1 


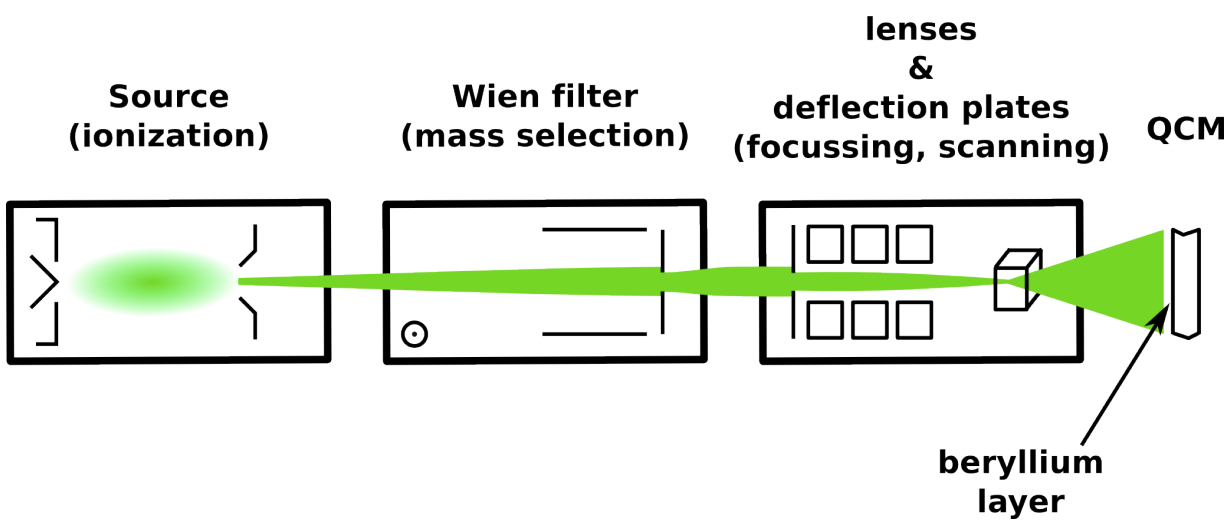

Figure 2 


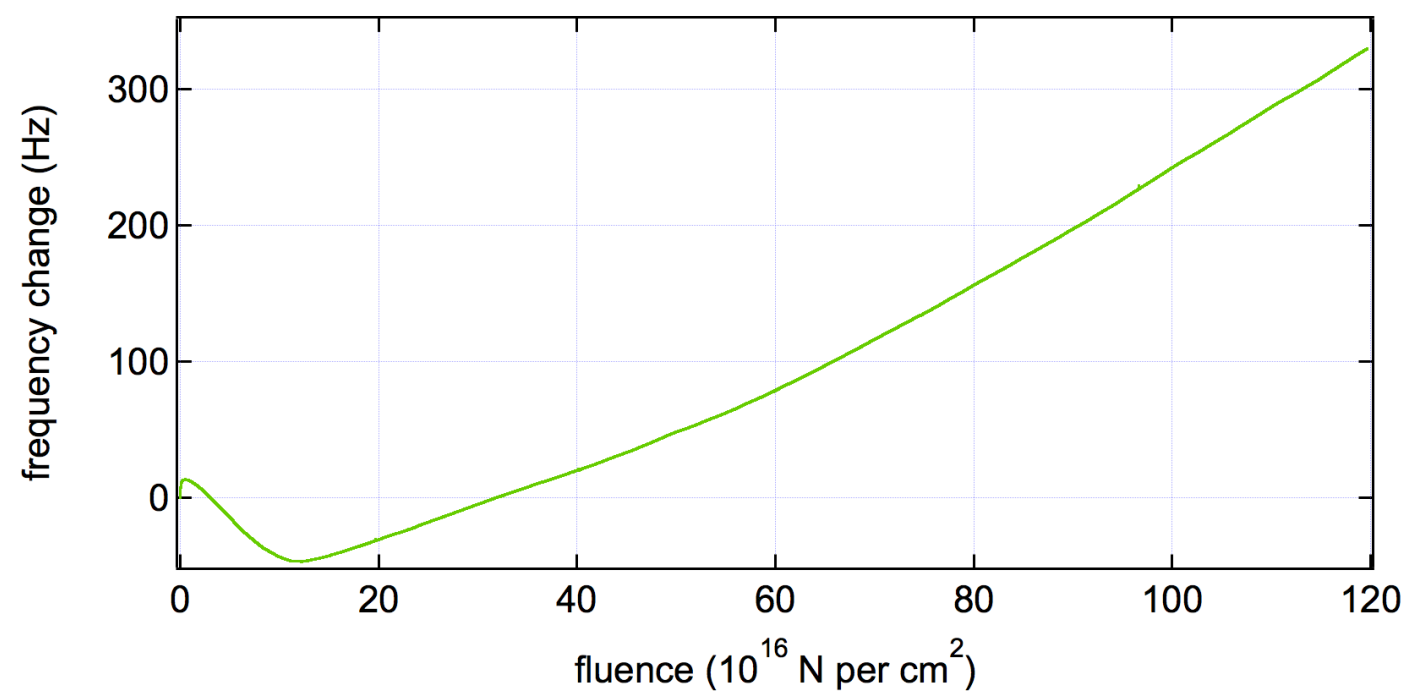

Figure 3 


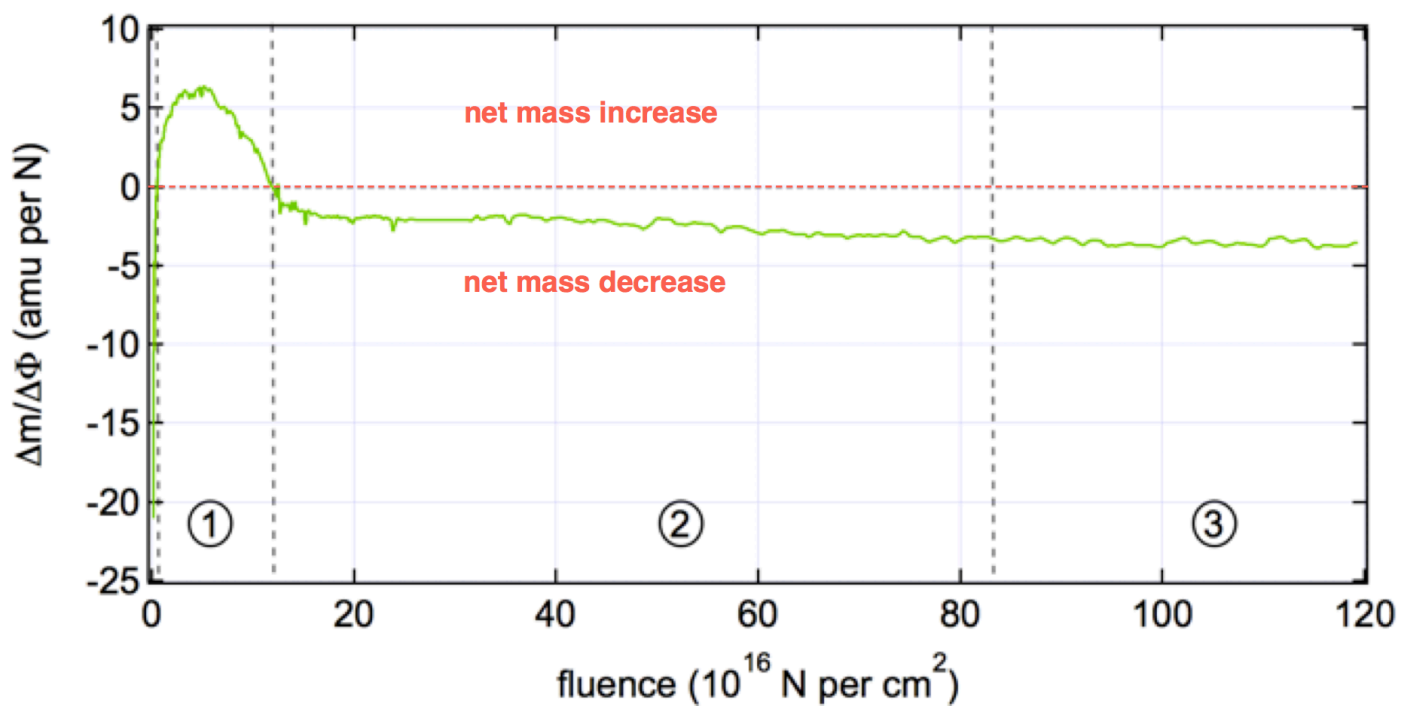

Figure 4 